\title{
Isolated Unilateral Vocal Fold Palsy following Skull Base Fracture: A Case Report
}

\author{
Marina $M B^{a}$, Hazleigh $N M^{b}$, Thean $Y K^{c}$, Sani $A .^{d}$ \\ a 'Department of Otorhinolaryngology, Faculty of Medicine, Universiti Kebangsaan Malaysia Medical Centre \\ ${ }^{b}$ Department of Otorhinolaryngology, Faculty of Medicine, Mara University Technology \\ ' Department of Radiology, Universiti Kebangsaan Malaysia Medical Centre \\ ${ }^{d}$ Department of Otorhinolaryngology, Faculty of Medicine, Universiti Kebangsaan Malaysia Medical Centre
}

\section{ABSTRACT}

Isolated lower cranial nerve $(\mathrm{CN})$ palsy affecting the $\mathrm{CN} X$ resulting from a skull base fracture is very rare. The clinical manifestation and natural history is related closely to the complex anatomy of this region and mechanism of injury. Here, we report a case of a 54 year-old man who presented with a delayed onset of dysphonia and dysphagia with aspiration following a closed head injury sustained from a motor vehicle accident. Injection laryngoplasty was implemented to alleviate symptoms of his CN X palsy, which eventually almost completely resolved. High index of suspicion should be maintained when investigating possible skull base fractures, especially with a suggestive clinical presentation of lower $\mathrm{CN}$ palsies affecting one or all the lower CNs. Delayed onset of these $\mathrm{CN}$ palsies are likely to have more favourable outcomes.

KEYWORDS: skull base fracture; cranial nerve; vocal fold palsy

\section{INTRODUCTION}

Lower cranial nerve $(\mathrm{CN})$ palsies resulting from skull base fractures are rarely reported in the literature. In the early $20^{\text {th }}$ century, Collet and Sicard had separately described a "pharyngeal scapular palateglossal laryngeal hemiplegia" following a gunshot injury. ${ }^{1}$

The degree of lower $\mathrm{CN}$ involvement varies, henceforth clinical syndromes have been described. Vernet syndrome is characterized by a lesion involving $\mathrm{CNs} \mathrm{IX}, \mathrm{X}$ and $\mathrm{XI}$, whereas a constellation of CN IX, X, XI and XII palsies is defined as ColletSicard syndrome. They are also known as jugular foramen syndrome and condylo-jugular syndrome respectively. ${ }^{1}$

Corresponding author:

Prof. Dr. Marina Mat Baki

Consultant Otorhinolaryngology

Level 9, Department of Otorhinolaryngology-Head and Neck Surgery

Universiti Kebangsaan Malaysia Medical Centre

Hospital Canselor Tunku Mukhriz

Jalan Yaakob Latif, Bandar Tun Razak

Cheras, 56000 Kuala Lumpur

Tel : 6019-653 4308

Email : marinamatbaki@ppukm.ukm.edu.my
We report a case of a skull base fracture resulting in an isolated $\mathrm{CN} X$ palsy, and discuss the diagnostic and therapeutic aspects of this case, and the relevant anatomy of this region in relation to its likely pathophysiology.

\section{CASE REPORT}

A 54-year-old man with no comorbidities was involved in a motor vehicle accident and was admitted to the local secondary care hospital for the initial management of his injuries, which included an intraparenchymal and subarachnoid haemorrhage, closed left radius and ulna fracture, and a compression fracture of the $1^{\text {st }}$ lumbar vertebra.

He presented to our tertiary centre with a chief complaint of clear watery rhinorrhoea aggravated by stooping, left unilateral hearing loss and a delayedonset of hoarseness with recurrent aspiration upon oral fluid intake three days following the initial trauma.

On examination, the patient had a full Glasgow Coma Scale score. His voice was breathy and there were no signs of upper airway obstruction. Clear fluid was noted to be leaking from the left 
sphenoethmoidal recess on nasal endoscopy and was suspicious of a cerebrospinal fluid (CSF) leak, which was confirmed biochemically. A flexible nasopharyngolaryngoscopy (FNPLS) demonstrated a left vocal fold paralysis with a large phonatory gap. Clinical hearing assessments were suggestive of a left profound sensorineural hearing loss, consistent with subsequent pure tone audiometry. All other CNs were intact. High-resolution computed tomograph (HRCT) of the base of skull and magnetic resonance imaging $(\mathrm{MRI})$ of the brain were performed. These imaging studies revealed the additional diagnosis of a skull base fracture involving the sphenoid sinus at its posterior wall and roof at the region of the pituitary fossa, the anterior ethmoid sinuses, petrous part of the temporal bone traversing it transversely, and extending into the left jugular foramen. [Figure 1, Figure 2].

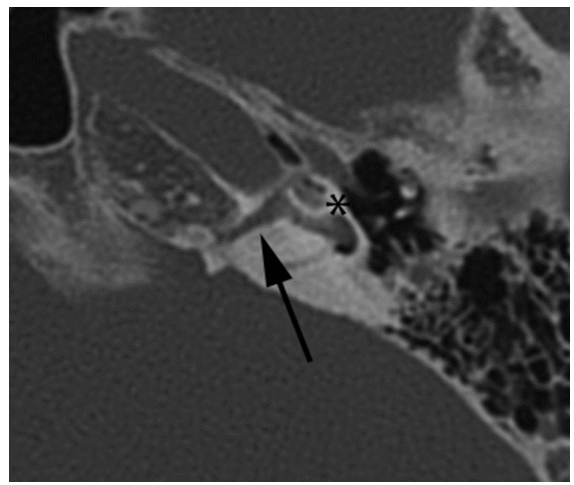

Figure 1 This figure shows an axial image of high resolution computed tomography (HRCT) of the left temporal bone at the level of the cochlear promontory. A transverse fracture involving the otic capsule is depicted (arrow) traversing the medial aspect of the cochlear basal turn. Subtle hyperdensity of the middle and basal turns of the cochlea $\left({ }^{*}\right)$ were of concern for labyrinthine ossification.

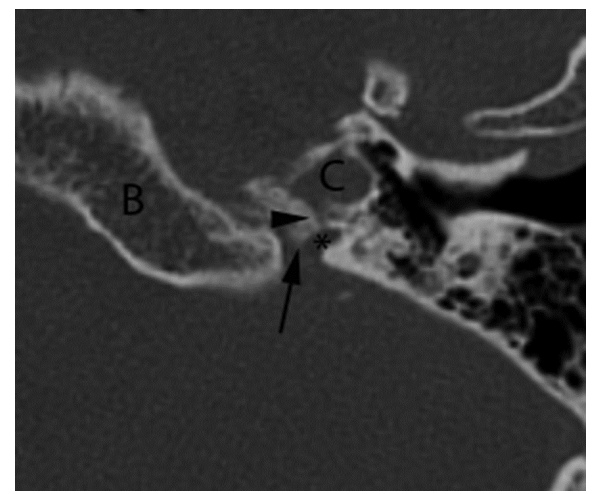

Figure 2 This figure shows an axial image of high resolution computed tomography (HRCT) of the left skull base at the level of the basisphenoid (B). The arrow points to the jugular spine. The inferior limit of the same fracture line (arrowhead) can be seen extending anteriorly from the osseous wall of the carotid canal (vertical segment, C), coursing posteriorly towards the pars vascularis of the jugular foramen $\left({ }^{*}\right)$
We proceeded with medialisation of the paralysed left vocal fold with a hyaluorinic acid injection laryngoplasty via direct laryngoscopy under general anaesthesia. It was done at the same sitting with endoscopic repair of the CSF leak.

The patient's immediate and early post-operative period was uneventful, with improvement of voice and resolution of aspiration hence oral feeding was recommenced. The CSF leak was also completely resolved.

Upon review in clinic, there was a significant improvement in the patient's dysphonia six months post-trauma, with mild residual breathiness. FNPLS confirmed return of function of the left vocal fold with a slight residual phonatory gap. Laryngeal mucosa sensation was intact with no signs of aspiration.

\section{DISCUSSION}

The hiatus formed between the petrosal part of the temporal bone and condylar part of the occipital bone ie the jugular foramen, conducts many vital structures between the cranial cavity and the neck. These include vascular structures such as sigmoid and inferior petrosal sinuses, jugular bulb, meningeal arteries from the ascending pharyngeal and occipital arteries. Also passing through the jugular foramen are the lower CNs: the glossopharyngeal ( $C N$ IX); vagus ( $C N X)$; and spinal accessory nerves (CN XI); and some of their smaller branches, such as Jacobson's nerve (branch from the $\mathrm{CN}$ IX) and Arnold's nerve (branch from the CN X). ${ }^{2}$

Traditionally, the jugular foramen is divided into two compartments: the smaller anteromedial pars nervosa containing the glossopharyngeal nerve and inferior petrosal sinus, and the larger posteriolateral pars vascularis containing the jugular bulb, medial to which are the vagus and spinal accessory nerves. ${ }^{2,3}$

These compartments are separated by bony projections from the temporal and occipital bones, the intrajugular spines, bridged by fibrous tissue of variable ossification collectively forming the intrajugular septum. The intrajugular septum has two characteristic dural perforations viz the glossopharyngeal and vagal meati that transmit the $\mathrm{CN}$ IX in the former, and $\mathrm{CN} X$ and $\mathrm{CN} X \mathrm{XI}$ in the latter. $^{2,3}$ 
The structures of the nerves are susceptible to palsies from fractures of the bones constituting this complex foramen, although other pathologies within this foramen or adjacent to it may also result in the typical constellation of lower $\mathrm{CN}$ palsies. This includes malignant lesions, which tend to be metastatic, and benign neoplasms such as glomus jugulare. ${ }^{4-6}$

The pathophysiology of lower $\mathrm{CN}$ palsies in base of skull fractures is related to direct mechanical trauma with early onset of palsies from compression or transection by displaced bony fragments and avulsion of the $\mathrm{CN}$ root, or neuropraxia, tissue oedema and the ensuing ischemia in cases where the palsies have a delayed onset. ${ }^{1,7,8}$

Lower $\mathrm{CN}$ palsies secondary to basal skull fractures are rare, however are more likely in fractures involving the occipital condyles. $31 \%$ of patients in a series of occipital condyle fractures reported by Tuli et al developed $\mathrm{CN}$ palsies, majority of which were of early onset. ${ }^{7}$

Imaging of choice for suspected base of skull fractures is a HRCT scan with an accompanying magnetic resonance (MR) scan, particularly when there are related lower $\mathrm{CN}$ palsies, to delineate these $\mathrm{CNs}$ as they pass through this vulnerable region. MR scans also aid in diagnosing related ligamentous injuries within the jugular foramen and extra-axial haemorrhages. Three-dimensional reconstruction from HRCT scans further improves the accuracy of diagnostic imaging of the skull base. $^{1,9}$

With the exception of coexistent cranio-cervical instability or CSF leaks, direct surgical treatment is not advisable and treatment is targeted at supportive measures, namely to alleviate dysphonia and aspiration. Authors did not report the use of steroids. ${ }^{2,8,10}$

To our knowledge, this case is only the second case of its nature to be reported in the literature ${ }^{10}$, however in contrast, the delayed onset of isolated $\mathrm{CN} X$ palsy and its near-complete resolution with supportive management suggests that the injury to this nerve was secondary to neuropraxia and oedema induced by the fracture traversing the pars vascularis.

\section{CONCLUSION}

Isolated $\mathrm{CN} \mathrm{X}$ palsies caused by skull base fractures are extremely rare. Supportive interventions such as hyaluronic acid injection laryngoplasty would be appropriate to provide symptomatic relief whilst allowing natural resolution of the underlying pathophysiological processes of oedema and neuropraxia.

\section{REFERENCES}

1. Legros B, Fournier P, Chiaroni P, Ritz O, Fusciardi J. Basal fracture of the skull and lower (IX, X, XI, XII) cranial nerve palsy: four case reports including two fractures of the occipital condyle a literature review. J Trauma. 2000; 48: 342-8.

2. Rothon AL. Jugular foramen. Neurosurgery 2000; 47 (Supplement): S267-S273.

3. Kanno T, Kiya N, Sunil MV. Microsurgical anatomy of the retroauricular, transcervico mastoid infralabyrinthine approach to jugular foramen. Neuroanatomy. 2003; 2: 28-34.

4. Prashant R \& Franks A. Collet-Sicard syndrome a report and review. Lanet Oncol. 2003; 4: 3767.

5. Khalid S, Zaheer S, Khalid M, Zaheer S, Raghuwanshi RK. Collet-Sicard syndrome secondary to a large glomus jugulotympanicum. Ann Saudi Med. 2013; 33(4): 407-10.

6. Smith R, Tassone P, Saada J. Collet-Sicard syndrome as a result of unilateral carotid artery dissection. BMJ Case Rep. July, 2013.

7. Tuli S, Tator $\mathrm{CH}$, Fehlings MG, Mackay M. Occipital condyle fractures. Neurosurgery, 1997; 41(2): 368-77.

8. Bozkurt G, Hazer B, Yaman ME et al. Isolated paralysis of glossopharyngeal and vagus nerve associated with type II occipital condyle fracture: case report. Childs Nerv Syst. 2010; 26: 719-22.

9. Laine FJ, Underhill T. Imaging of the lower cranial nerves. Magn Reson Imaging Clin N Am. 2002; 10: 433-449.

10. Huang $H$, Fang $T$, Li H, Chin S. Vagus nerve paralysis due to skull base fracture. Auris Nasus Larynx. 2008; 35: 153-5. 
